\title{
Dynamic Fracture Model for Acoustic Emission
}

\author{
Manuela Minozzi, ${ }^{1,2,3}$ Guido Caldarelli, ${ }^{1}$ Luciano Pietronero, ${ }^{1,3}$ and Stefano Zapperi ${ }^{1}$ \\ ${ }^{1}$ INFM UdR Roma 1 - Dipartimento di Fisica, Università "La Sapienza", P.le A. Moro 2, 00185 - Roma, Italy \\ ${ }^{2}$ INFM UdR Roma 3, Dipartimento di Fisica, Università di Roma 3, via della vasca navale 84, 00146 Roma, Italy. \\ ${ }^{3}$ CNR Istituto di Acustica "O. M. Corbino", via del fosso del Cavaliere 100, 00133 Roma, Italy
}

(Dated: August 14, 2021)

\begin{abstract}
We study the acoustic emission produced by micro-cracks using a two-dimensional disordered lattice model of dynamic fracture, which allows to relate the acoustic response to the internal damage of the sample. We find that the distributions of acoustic energy bursts decays as a power law in agreement with experimental observations. The scaling exponents measured in the present dynamic model can related to those obtained in the quasi-static random fuse model.

PACS numbers: $62.65 .+\mathrm{k}, 46.50 .+\mathrm{a}, 63.70 .+\mathrm{h}$
\end{abstract}

Crackling noise [1] is widely observed in systems as different as superconductors [2], magnets [3] or plastically deforming crystals 泪. A typical example is the acoustic emission (AE) recorded in a stressed material before failure. The noise is a consequence of micro-cracks forming and propagating in the material and should thus provide an indirect measure of the damage accumulated in the system. For this reason, AE is often used as a nondestructive tool in material testing and evaluation. Beside these practical applications, understanding the statistical properties of crackling noise has become a challenging theoretical problem. The distribution of crackle amplitudes follows a power law, suggesting an interpretation in terms of critical phenomena and scaling theories. This behavior has been observed in several materials such as wood [5], cellular glass [6], concrete [7] and paper [8] to name just a few.

The statistical properties of fracture in disordered media are captured qualitatively by lattice models, describing the medium as a discrete set of elastic bonds with randomly distributed failure thresholds [9, 10, 11]. After each crack the stress is redistributed in the lattice in a quasi-static approximation: i.e. the crack velocity is much slower than stress relaxation. Thus acoustic waves are not taken into account and the activity is monitored by the damage evolution or by the dissipated elastic energy. Numerical simulations indicate that microcracks propagate in avalanches giving rise to an heterogeneous response. The avalanche distribution is typically described by power law distributions and the results are usually interpreted in the framework of phase transitions [12, 13, 14, 15, 16]. Despite the fact that critical phenomena are normally associated with a certain degree of universality (i.e. the scaling exponents should not depend on micro-structural details), there has been so far no quantitative agreement between models and experiments. A reason that could account for this discrepancy is the absence of acoustic waves in most models. It is then not obvious how to relate AE activity to internal avalanches.

Dynamic lattice models have been widely used in the past to analyze fracture processes [17, 18, 19, 20], but although acoustic waves are explicitly included, the AE sig- nal is usually not analyzed. Here we use a lattice model for dynamic fracture in a disordered medium, to obtain a direct correspondence between the recorded $\mathrm{AE}$ activity and the internal damage evolution. We find that the cumulative $\mathrm{AE}$ amplitudes are directly related - by a power law - to the cumulative damage. Next, we measure the distribution of the $\mathrm{AE}$ burst energies and find a power law with an exponent $\beta \simeq 1.7$ independent on the loading rate. This exponent can be related to the exponent describing failure avalanches in quasi-static models 112, 13, 14, 15, 16.

We consider a scalar model of dynamic fractures where a two-dimensional lattice is loaded in mode III [18]: the lattice lies in the $(x, y)$ plane and deformation occurs along the $z$ axis, so that the equations of elasticity become scalar. The equation of the motion for the antiplanar displacement $u$ of a site with coordinate $i, j$ is

$$
\rho \ddot{u}_{i, j}=-K \sum_{(l, m)}\left(u_{i, j}-u_{l, m}\right)-\Gamma \dot{u}_{i, j}
$$

where the sum runs over the nearest neighbors $(l, m)$ of site $(i, j), K$ is the elastic constant, $\rho$ is the density and dissipation is simulated by a viscous damping with a constant $\Gamma$. In order to suppress some lattice effects, we use a 45 degree tilted square lattice. A constant strain rate is imposed to the model, by moving the boundary sites on two opposite boundaries at constant velocity $V$ and $-V$, respectively. Periodic boundary conditions are imposed in the other direction. Disorder is simulated assigning randomly distributed failure threshold: a bond is removed (i.e. $K$ is set to zero) when $\Delta u>f_{c}$, and $f_{c}$ is uniformly distributed in $[0,1]$. Notice that in the quasistatic limit $(V \rightarrow 0, \rho \rightarrow 0, \Gamma \rightarrow 0)$ the model reduces to the random fuse model (RFM), where a lattice of fuses with random threshold are subject to an increasing voltage [10, 11]. Due to the scalar nature of our model there is a direct mapping between elastic and electric parameters 9].

The equation of motion (Eq. 1) is integrated numerically using a fourth order Runge-Kutta method. We work with a lattice of linear size $L=80$ and chose the units of space and time so that $\rho=K=1$. Each time 


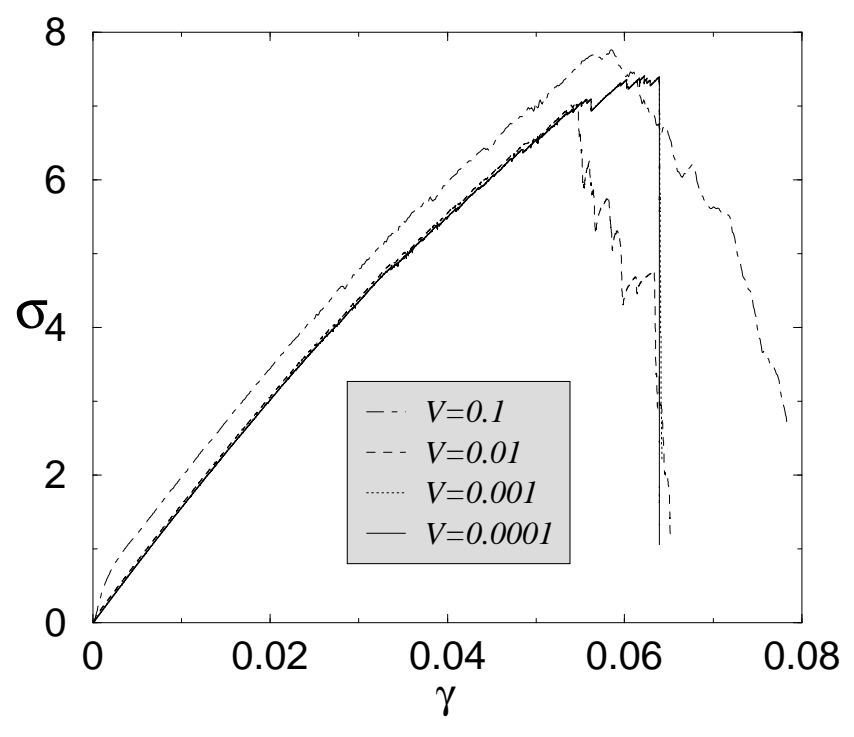

FIG. 1: The stress-strain curve for different applied strain rates.

a bond is stretched beyond its threshold the lattice constant is set to zero and an elastic wave is emitted. Due to the anti-plane constraint for the displacements, we only have transverse wave propagation with sound speed $c=\sqrt{K / \rho}=1$ in our units. The damping constant is chosen to be $\Gamma=0.1$ so that typical length traveled by a wave is a little smaller than the lattice size. For smaller values of $\Gamma$ ringing effects and reflected waves do not allow to separate the single pulses and the lattice breaks at once. On the other hand, excessive damping leads to very small acoustic activity and the sample breaks suddenly at the edges. Even if the damping constant is small reflected waves can induce boundary failure, due to the rigidity of the loaded edge. Thus we do not allow that bonds fail in two boundary layers of length $l=5$ close to the loaded edges. This corresponds to apply a load through a soft contact. The model is simulated for a variety of loading velocities all much lower than the sound speed $V \ll c$.

Measuring the displacements of every lattice site and calculating the forces for every time steps, we have obtained the stress-strain curve for four different value of the applied strain rate. In Fig. 11 we show that the stress is a linear function of the strain up to the yield point, which precedes the total failure of the sample. The applied strain rate has little effect on the linear part of the curve, while it influences the curve after the yield point.

Monitoring the activity of some particular lattice sites we have direct access to the AE signal. These sites mimic the effect of transducers coupled to the material in a typical $\mathrm{AE}$ experiment. In a typical run, we record the displacements, velocities and accelerations of four sites in the boundary layer and two sites in the interior. Typically, AE distributions are recorded from a single site and averaged over ten realizations of the disorder. We have tested that the statistical properties of the signal do not vary for different boundary sites, while there is a clear difference between boundary and inner sites. In the following, we concentrate on sites in the boundary layer, in order to avoid excessive fluctuations due to failures occurring on neighboring bonds in the inner region.

An example of the typical signals recorded with our model are reported in Fig. 2. A large acoustic activity is visible in the upper panel where we show the local acceleration $a$ of a boundary site as a function of time. We can also monitor the velocity signal which is simply related to the acceleration and display the same features. In the present model, it will be convenient to use the acceleration as a $\mathrm{AE}$ monitoring tool, since the velocity has a bias induced by the external loading: even in the absence of cracking the lattice has a non-vanishing velocity. We define the associated cumulative energy as

$$
E(t) \equiv \int_{0}^{t} d t^{\prime} a^{2}\left(t^{\prime}\right)
$$

The behavior of the cumulative acoustic energy $E(t)$ is typically monitored in $\mathrm{AE}$ experiments. In some cases, $E(t)$ is found to increase as a power law [5], or exponentially in other cases [8]. In general one expects a marked peak close to failure, as we also observe in Fig 2 , obtained for $V=10^{-3}$. The curve is well fitted by cubic law, $E \sim t^{3}$.

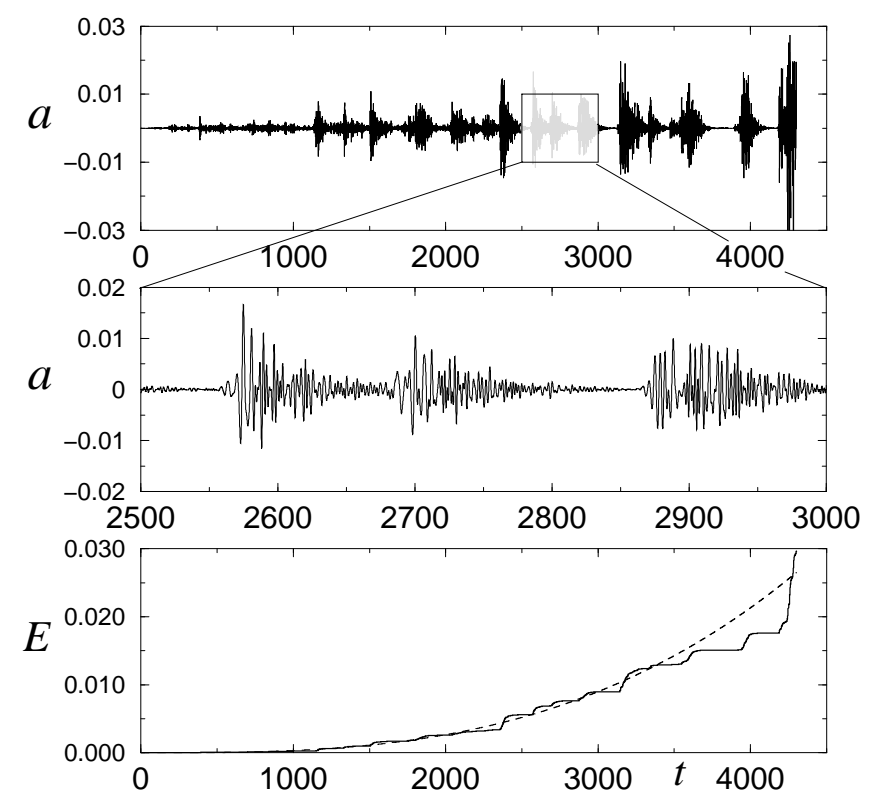

FIG. 2: A typical signal measured in the model. The upper panel shows the velocity acceleration of a site close to the boundary. In the middle panel we show a magnification of a portion of the signal. In the lower panel is reported the cumulative energy, $E(t) \equiv \int_{0}^{t} d t^{\prime} a^{2}\left(t^{\prime}\right)$, as a function of time for a single realization of disorder. The dashed line follows $t^{3}$.

A central problem in AE measurements is to correlate the recorded acoustic activity with the internal damage 


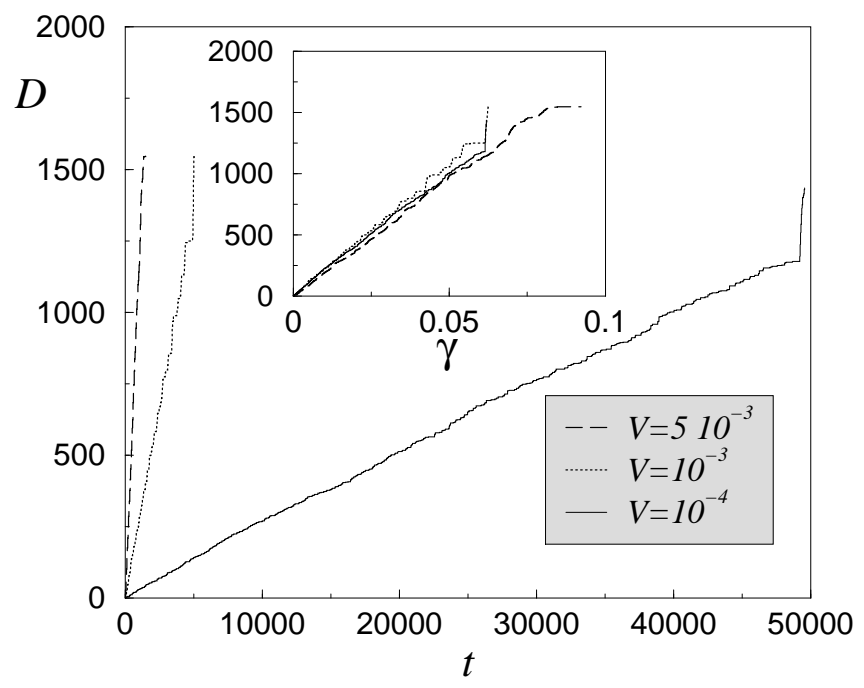

FIG. 3: The damage $D$ evolution is displayed as a function of time for different loading rates. The curves collapse onto each other when plotted with respect to strain $\gamma \equiv V t / L$.

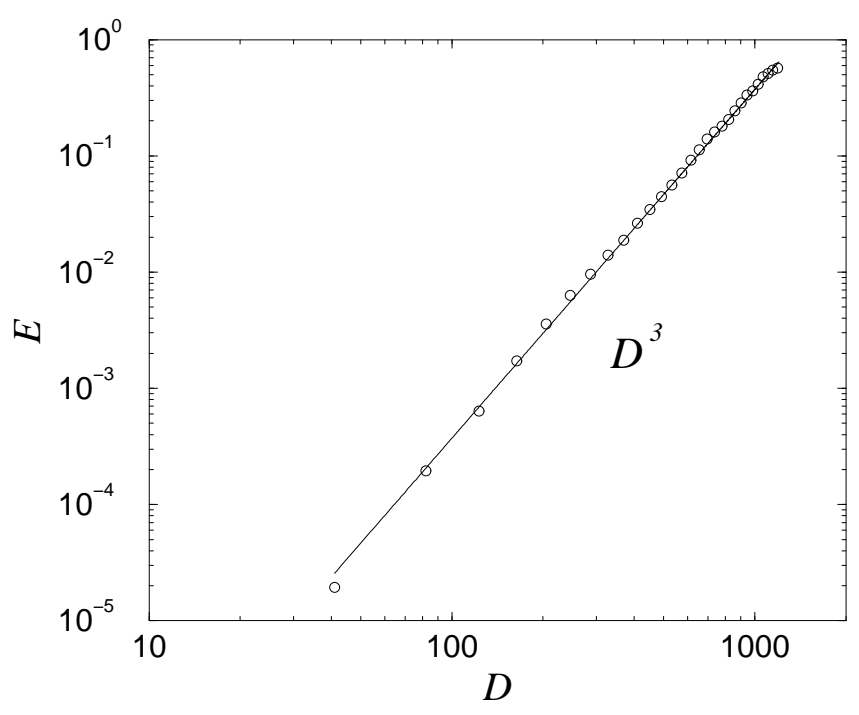

FIG. 4: The average cumulative energy plotted as a function of damage. The line represents a $D^{3}$ law which fits well the curve.

state. In this way, AE can be used as a tool for damage evaluation. In our model, we have a direct access to the internal damage $D$ that can be defined as the total number of failed bonds. We find that $D$ increases linearly with time (see Fig. 3) apart from a rapid increase very close to failure. Rescaling the curves with the loading rate one sees that $D$ is in fact a linear function of the applied strain $\gamma \equiv(V t) / L$ (see the inset of Fig. - 4 ).

These observations thus lead to a direct scaling relation between internal damage and released acoustic energy: Fig. 1 shows that $E$ scales as expected as $D^{3}$. A direct consequence of this result is that the measured acoustic

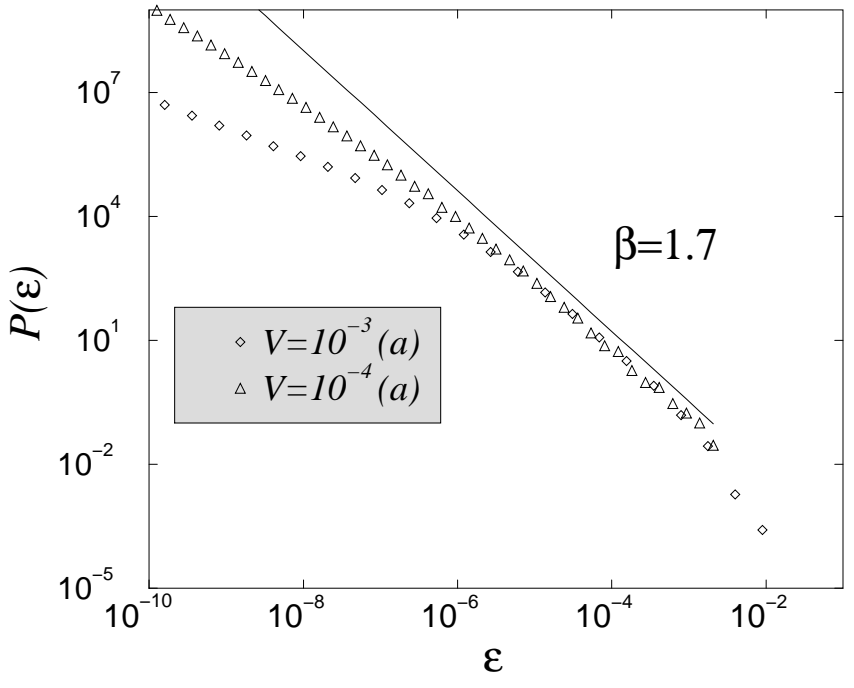

FIG. 5: The distribution of acoustic burst energies for different driving rates. The line is a power law with exponent $\beta=1.7$.

energy is proportional to the released elastic energy $E_{e l} \sim$ $K D \gamma^{2} \sim D^{3} \sim E$.

A large amount of theoretical activity has been devoted in the past to understand the origin of power law distributions of $\mathrm{AE}$ amplitudes widely observed in material fracture. Most of the analysis was devoted to quasi-static models, such as the the RFM, where fracture was shown to occur in damage burst, distributed as $P(D) \sim D^{-\tau}$ with $\tau \simeq 2.5[12,13$. This value is in perfect agreement with the result $\tau=5 / 2$ obtained exactly 21] for the exponent of the avalanche distribution of the fiber bundle model (FBM) [22], where $N$ fibers with random failure threshold are loaded in parallel. It was thus conjectured that the long-range stress transfer present in the RFM was equivalent to the infinite range load redistribution of the FBM, placing the two models into the same universality class 12, 13. A similar exponent was found in a vectorial fracture model, so that this class could be even broader 13. Comparing this result with $\mathrm{AE}$ experiments is problematic since quasi-static models do not account for wave propagation.

Here we can directly measure the distribution of pulse sizes due to the acoustic activity. In Fig. 0 we report the distribution of energies for the acceleration signal, defining $\epsilon \equiv a^{2}$. In both cases the distribution decays as power law with an exponent $\beta=1.7 \pm 0.1$, independent on the loading rate, which only affects the low part of the distribution. The same law is found in the case of the velocity signal. Experimental results report an exponent value in the same range, even if it differs a little from one material to another: for wood the exponent is $\beta=$ $1.51 \pm 0.05$, for fiberglass $\beta=2.0 \pm 0.01$ [5], $\beta=1.30 \pm 0.1$ for paper [8], $\beta=1.5 \pm 0.1$ for experiments on cellular glass [6].

Using the scaling relation between released acoustic 
energy and damage discussed above, we can relate the exponent $\beta$ to $\tau$. From $E \sim D^{3}$ and $D \sim t$, we expect $\epsilon \sim D^{2}$. Substituting this expression in the equation for the probabilities $P(\epsilon) d \epsilon=P(D) d D$, we obtain $\tau=1+2(\beta-1)=2.4$, which is very close to $\tau=5 / 2$ measured in the RFM. Thus we conjecture that the acoustic energy exponent measured in our dynamic model is directly related with the damage exponent measured in the corresponding quasi-static model [23].

In conclusions, we have introduced a lattice model of dynamic fracture which can be used to model AE experiments. The model allows to clarify important issues in the interpretation of the experiments, namely the relation between internal damage and released acoustic en- ergy. In particular, we derive direct relations between the scaling behavior of failure avalanches and acoustic bursts. It would be interesting to generalize this analysis to more realistic situations, exploring the role of dimensionality, load conditions and lattice anisotropy. However, in comparing the simulated signal with experiments, we should be careful about the definition of the events in the time series, since the amplifier and the AE sensors could bias the recorded waveform, introducing a systematic error in the data.

This work has been supported by the European Network contract FMRXCT980183 and the INFM center SMC.
[1] J. Sethna, K. A. Dahmen, C. R. Myers, Nature 410242 (2001).

[2] S. Field, J. Witt, F. Nori and X. Ling, Phys. Rev. Lett. 74, 1206 (1995).

[3] G. Durin and S. Zapperi, Phys. Rev. Lett. 84, 4705 (2000).

[4] M. C. Miguel, A. Vespignani, S. Zapperi, J. Weiss and J. R. Grasso, Nature 410, 667 (2001).

[5] A. Garcimartín, A. Guarino, L. Bellon and S. Ciliberto, Phys. Rev. Lett. 79, 3202 (1997); A. Guarino, A. Garcimartín and S. Ciliberto, Eur. Phys. J. B 6, 13 (1998).

[6] C. Maes, A. Van Moffaert, H. Frederix and H. Strauven, Phys. Rev. B 57, 4987 (1998).

[7] A. Petri, G. Paparo, A. Vespignani, A. Alippi and M. Costantini, Phys. Rev. Lett. 73, 3423 (1994).

[8] L.I. Salminen, A.I. Tolvanen, and M. J. Alava, Acoustic Emission from Paper Fracture, preprint.

[9] H.J. Herrmann and S. Roux (eds.), Statistical Models for the Fracture of Disordered Media (North Holland, Amsterdam, 1990).

[10] L. de Arcangelis, S. Redner and H. J. Herrmann, J. Phys. Lett. (Paris) 46, L585 (1985).

[11] P. M. Duxbury, P. D. Beale and P. L. Leath, Phys. Rev. Lett. 57, 1052 (1986).

[12] A. Hansen and P. C. Hemmer, Phys. Lett. A 184, 394 (1994).

[13] S. Zapperi, P. Ray, H. E. Stanley, and A. Vespignani,
Phys. Rev. Lett. 78, 1408 (1997); Phys. Rev. E 59, 5049 (1999).

[14] S. Zapperi, A. Vespignani, and H. E. Stanley, Nature (London) 388, 658 (1997).

[15] G. Caldarelli, F. D. Di Tolla and A. Petri Phys. Rev. Lett. 77, 2503 (1996).

[16] V. I. Räisänen, M. J. Alava, R. M. Nieminen, Phys. Rev. B, 58, 14288 (1998).

[17] See J. Fineberg and M. Marder, Phys. Rep.313, 1 (1999), for a review on dynamic fracture.

[18] M. Marder and X. Liu, Phys. Rev. Lett. 71, 2417 (1993).

[19] T. T. Rautiainen, M. J. Alava, and K. Kaski, Phys. Rev. E 51 R2727 (1994); ibid 56, 6443 (1997).

[20] T. Martín, P. Español, M. A. Rubio, and I. Zúñiga Phys. Rev. E 61, 6120 (2000)

[21] P. C. Hemmer and A. Hansen, J. Appl. Mech. 59, 909 (1992); M. Kloster, A. Hansen and P. C. Hemmer Phys. Rev E 56, 2615 (1997).

[22] H. A. Daniels, Proc. Roy. Soc. London A183, 405 (1945); S. L. Phoenix and H. M. Taylor, Adv. Appl. Prob. 5, 200 (1973).

[23] The distribution of the "elastic" energy released in each avalanche was recently measured in the quasi-static FBM and found to decay as power law with exponent $\beta \simeq 1.8$ [8], in agreement with our results. 